\title{
A Rare Presentation of Transvaginal Septum in Second Trimester Abortion: A Case Report
}

\author{
Sium AF*, Tilahun A, Mersha A, Yihun S and \\ Shiferaw MA \\ Department of Obstetrics and Gynecology, Saint Paul's \\ Hospital Millennium Medical College, Ethiopia \\ *Corresponding author: Abraham Fessehaye Sium, \\ Department of Obstetrics and Gynecology, Saint Paul's \\ Hospital Millennium Medical College, Addis Ababa, \\ Ethiopia
}

Received: February 03, 2021; Accepted: February 25, 2021; Published: March 04, 2021

\begin{abstract}
Background: A transvaginal septum occurs if these two tissue groups do not fuse properly by 5 months of embryonic life. The recommended mode of safe termination for second trimester pregnancy with a diagnosis of high transverse vaginal septum is cervical preparation followed by septostomy and dilation and curettage.
\end{abstract}

Case Summary: A 28 years-old gravida-II Para-I (alive by CS for unknown indication) presented at gestational age 15 weeks plus 6 . Hysterotomy was done for an indication of septic inevitable missed second trimester abortion plus presumptive diagnosis of high vaginal septum.

Conclusion: According to the case reports that has been reported, the recommended management for safe termination of second trimester pregnancy with a diagnosis of transvaginal septum is good cervical preparation followed by septostomy and dilation and curettage (D\&E).

Keywords: Transverse vaginal septum; Second trimester abortion; Dilation and curettage

\section{Background}

A transvaginal septum or cervical agenesis occurs if these two tissue groups do not fuse properly by 5 months of embryonic life. The most common location for the septum is in the upper third of the vagina, and only $10 \%$ of cases are imperforate. The recommended mode of safe termination for second trimester pregnancy with a diagnosis of high transverse vaginal septum is cervical preparation followed by septostomy and dilation and curettage.

\section{Case Presentation}

A 28 years-old gravida-II Para-I (alive by CS for unknown indication) whose gestational age from reliable date was 15 weeks plus 6 days admitted for safe termination of pregnancy with a diagnosis of inevitable second trimester missed abortion plus septic abortion to rule out pyelonephritis. She presented with a history of passage liquor of 17 hours duration and associated history of fever, lower abdominal pain, and vaginal bleeding of one-day duration. She was put on intravenous antibiotics - ceftriaxone plus metronidazole. And medication abortion was started.

After she took five doses of 400 micrograms misoprostol sublingual, there was no progress of cervical dilation up on subsequent evaluation. She had tachycardia of 120 beats per minute and there was low-grade fever of 38.8 degree centigrade. On speculum exam, we were unable to identify the cervix, noting smooth vaginal tissue throughout. There was a small pinhole orifice in the upper vagina, which was oozing blood. On bimanual exam, cervix was palpated behind a wall of tissue, suspicious for transverse vaginal septum. Bedside ultrasound was done but we were unable to pick the transvaginal septum.

With a diagnosis of septic missed second trimester abortion plus presumptive diagnosis of high vaginal septum, hysterotomy was done which evacuated 100 grams abortus from the uterine cavity. Remnants of conceptus tissue along with clots of blood was also extracted from the vaginal cavity, while we confirmed the diagnosis of high-transverse vaginal septum intra-operatively.

Our patient had smooth post-operative course. She was discharged after three days of parenteral antibiotics treatment. Her subsequent follow-up visit was after one week and it did not document any abnormality. She had good wound healing. An elective cesarean delivery was decided for her future birth plan and she was well counselled about it.

\section{Discussion}

Normal vaginal development requires the downward growing Müllerian ducts to fuse with the upward-growing derivative of the urogenital sinus [1]. A transvaginal septum or cervical agenesis occurs if these two tissue groups do not fuse properly by 5 months of embryonic life. The most common location for the septum is in the upper third of the vagina, and only $10 \%$ of cases are imperforate [2]. Matching this truth in our case the diagnosis was a high tranvaginal septum.

Patients with an imperforate transverse vaginal septum typically present in adolescence with primary amenorrhea and require surgical correction. Pregnancy in these women is associated with a high spontaneous abortion rate [3]. Our case presented as a septic inevitable missed second trimester abortion.

Gibson reported a case of spontaneous abortion at 13 weeks' gestation, in which the POCs became entrapped between the cervix and a transverse septum. He dilated the septum to $6 \mathrm{~mm}$ with Hegar dilators and removed the POCs with Desjardin forceps; the septum 
was resected at a later date [4].

In 2017, Jennifer A Pitotti reported a case, which involved a woman seeking an elective termination at 16 weeks' gestation, and resection of the septum was required in order to access the cervix and perform the uterine evacuation [5]. Taking into consideration the seriousness of her diagnosis of septic abortion, immediate hysterotomy was done in our case to facilitate evacuation after a failed attempt of medication abortion.

\section{Conclusion}

According to the case reports that has been reported, the recommended management for safe termination of second trimester pregnancy with a diagnosis of transvaginal septum is good cervical preparation followed by septostomy and dilation and curettage. This recommendation was the ideal one for our case. However; taking the amount of time that would be required to prepare the cervix further and the seriousness of septic abortion (which could have progressed to generalized sepsis) into consideration, we decided to do hysterotomy on time instead of waiting.

\section{Declarations}

\section{Consent for publication}

A verbal informed consent was obtained from the patient for publication of this case.

\section{Availability of supporting data}

All supporting documents are submitted along with the case report

\section{Authors' contributions}

$\mathrm{AF}$ and MA contributed the introduction and case summary. $\mathrm{AM}, \mathrm{AT}$, and SY prepared the discussion and conclusion part.

\section{Funding}

This research did not receive any specific grant from funding agencies in the public, commercial, or not-for-profit sectors.

\section{Acknowledgements}

The authors would like to thank the Department of Obstetrics and Gynecology at Saint Paul's Hospital millennium Medical College, Addis Ababa, Ethiopia.

\section{References}

1. Rock JA. Surgery for anomalies of the molarium ducts. In Rock JA, Thompson JD, eds. TeLinde's Operative Gynecology. Philadelphia: JB Lippincott. 1997: 687.

2. Rock JA, Zacur HA, Dlugi AM, Jones HW, TeLinde RW. Pregnancy success following surgical correction of imperforate hymen and complete transverse vaginal septum. Obstet Gynecol. 1982; 59: 448-451.

3. Fritz EB, Carlan SJ, Greenbaum L. Pregnancy and transvaginal septation, The Journal of Maternal-Fetal \& Neonatal Medicine. 2002; 11: 414-416.

4. Gibson ED. Transverse upper vaginal septum presenting in pregnancy: a case report and review of the literature. Aust N Z J Obstet Gynaecol. 2003; 43: 381-383.

5. Jennifer AP, Veronica IA, Stephanie T, Kristina T. Transverse vaginal septum identified in a patient requesting second trimester abortion: a case report. Contraception. 2017. 